\title{
Mid-term results after proximal humeral fractures following angular stable plate fixation in elderly patients - which scores can be evaluated by a telephone-based assessment?
}

Patrick Ziegler ${ }^{1}$, Kim Stierand $^{1}$, Christian Bahrs ${ }^{1 *}$ and Marc-Daniel Ahrend ${ }^{1,2}$

\begin{abstract}
Background: The aim was to evaluate postsurgical outcome in elderly patients ( $>70$ years) after open reduction and internal fixation (ORIF) of proximal humeral fractures and compare the test-retest agreement of scores which are frequently used to assess the outcome of upper extremity disorders.

Methods: Ninety patients ( $78.1 \pm 5.2$ years) with a minimum follow-up of 2 years ( $3.7 \pm 0.9$ years) following angular stable plate fixation of a proximal humeral fracture (2-part: 34, 3-part: 41, 4-part: 12) were enrolled. Two telephonebased interviews assessed Disabilities of the Arm, Shoulder and Hand Score (DASH), Oxford Shoulder Score (OSS), and Constant Score adjusted for interview assessment (CS) by two independent interviewers. Correlations, BlandAltman analyses, Cross tabulation, and weighted Kappa measure of agreement ( $k$ ) were calculated to assess differences and the test-retest agreement between the categories of each score.

Results: In the first and second interview, we could state fair outcomes: CS 91 (range 40-100) and 65.5 (23-86), DASH $12.5(0-64.2)$ and $18.3(0-66.7)$, and OSS 58 (33-60) and 55 (25-60) points.

The test-retest correlations were $r=0.67, r=0.77$, and $r=0.71$ for CS, DASH, and OSS. Bland-Altman analyses showed absolute mean individual score differences of $-22.3,4.9$, and -3.0 for CS, DASH, and OSS. Limits of agreement represented possible differences of $21.6 \%, 15.5 \%$, and $9.0 \%$ of CS, DASH, and OSS. The category agreements were medium to high: CS 55.9\% ( $k=0.08)$, DASH 87.2\% $(k=0.62)$, and OSS 99.3\% $(k=0.74)$.
\end{abstract}

Conclusion: Patients showed good subjective outcomes. The test-retest agreement of the interview-adjusted CS was low, but telephone-based assessment of OSS and DASH present as an alternative to collect outcomes in elderly patients.

Trial registration: (250/2011BO2).

Keywords: Angular stable plate fixation, Proximal humeral fracture, Fractures in the elderly, Outcome

\footnotetext{
*Correspondence: c.bahrs@gmx.de

${ }^{1}$ BG- Center for Trauma \& Reconstructive Surgery, Eberhard Karls University of

Tübingen, 72076 Tübingen, Germany

Full list of author information is available at the end of the article
}

(c) The Author(s). 2020 Open Access This article is distributed under the terms of the Creative Commons Attribution 4.0 International License (http://creativecommons.org/licenses/by/4.0/), which permits unrestricted use, distribution, and reproduction in any medium, provided you give appropriate credit to the original author(s) and the source, provide a link to the Creative Commons license, and indicate if changes were made. The Creative Commons Public Domain Dedication waiver (http://creativecommons.org/publicdomain/zero/1.0/) applies to the data made available in this article, unless otherwise stated. 


\section{Background}

Proximal humeral fractures are common injuries of the elderly patient with an increasing incidence [1-4]. Depending on factors such as patient age, pre-existing conditions, degree of dislocation, fracture morphology, and patient's expectation, the decision between conservative treatment and surgical intervention is made. However, no consensus about the gold-standard treatment of proximal humeral fractures is present [5-7]. Especially in patients with a multifragmentary fracture and/or severe fracture dislocation, open reduction and internal fixation is often the treatment of choice [8-10]. Overall good results in 70 to $80 \%$ of the cases can be achieved with angular stable plates. However, complications such as screw perforation, humeral head necrosis, and secondary fracture displacement are common [11, 12]. So far, long-term results following angular stable plate fixation for proximal humeral fractures are limited in the literature, especially for elderly patients [13-15]..

Patient-reported outcome measures (PROM) are important evaluation tools to assess clinical and functional outcomes from the patient's perspective $[16,17]$. The measurement of surgical outcome parameters and the detection of complications is difficult in elderly trauma patients, because follow-up appointments often represent a barrier for this patient group with immobility and dependency of assisted transportation. Therefore, telephone-based assessments of PROMs can be a timesaving alternative in this patient group. However, no data is available regarding the usefulness as well as the test-retest agreement of telephone-based assessment of standardized scores in proximal humeral fractures of elderly patients.

This cohort study had the primary aim to assess midterm results using standardized and established scores (DASH Score-Disabilities of the Arm, Shoulder and Hand), OSS (Oxford Shoulder Score), and the interviewbased Constant score according to Boehm at al [18]. in patients aged over 70 years with proximal humeral fractures treated with angular stable plate fixation. The secondary aim was to compare the test-retest agreement of the telephone-based assessment of the scores. We hypothesized (1) that the majority of patients had good results at least 2 years after surgery in all three scores and (2) that the DASH and the OSS had higher test-re-test agreement compared to the Constant score regarding the subjective shoulder function in the elderly population.

\section{Materials and methods}

\section{Study design and patient recruitment}

The present cohort study (level IV) analyzed functional surgical outcomes of proximal humeral fractures with angular stable plate fixation at least 2 years postoperatively and was approved by the local ethics committee. Inclusion criteria were a minimum age of 70 years and a proximal humeral fracture following ORIF. Exclusion criteria were change of therapeutic concept of an anatomical reconstruction of the humeral head during the follow-up period (e.g., revision surgery with arthroplasty) and non-shoulder-related severe comorbidities (e.g., dementia). Within a 2-year timeframe, 160 consecutive patients with proximal humeral fractures older than 70 years were treated with angular stable plate fixation. All patients were contacted at least 2 years ( $3.7 \pm 0.9$ (range 2.3 to 5.4 years) following surgi$\mathrm{cal}$ intervention by written letter for study participations 2 weeks ahead of telephone-based interviewing. A second interview-based score assessment was performed 3 months after the first interview within a 2-week period. The first interview was conducted by an experienced physician assistant. The second interview was conducted by a medical doctor (resident). Both interviewers were blinded about the pre-, peri- and postoperative details and were not involved in the surgeries of the patients. Both interviewers were experienced in data collection for research projects more than 3 years. Both interviewers were educated by the same senior consultant to have higher interview standardization. The patient flow chart is presented in Fig. 1. The work has been reported in line with the STROCSS criteria [19].

\section{Participants}

The final data set comprised 90 patients (male: 12, female: 78 ; height: $164.8 \pm 7.2 \mathrm{~cm}$; weight: $68.9 \pm$ $17.8 \mathrm{~kg}$ ) with a mean age of $78.1 \pm 5.2$ years (range 70.1 to 89.8 years). According to the Neer classification [20], the cohort consisted of 34 2-part fractures, 41 3-part fractures, and 12 4-part fractures. According to the AO/OTA classification [21], these fractures were classified as $34 \mathrm{~A}, 32 \mathrm{~B}$, and $21 \mathrm{C}$ fractures. No traumatic nerve injury or vascular comorbidities occurred. Fractures were treated in $28.9 \%$ of the cases with a PHILOS ${ }^{\circ}$ plate (DePuy Synthes, West Chester, Pennsylvania, USA) and in $71.1 \%$ of the cases with a WINSTA-PH WS proximal Humerus (Axomed GmbH, Freiburg, Germany).

\section{Surgical treatment and postoperative care}

Patients were treated using either the PHILOS $^{\circ}$ plate or the WINSTA-PH WS ${ }^{\circ}$ proximal humeral plate. The operations were performed by using either a deltoid split, a deltoideo-pectoral, or an anterolateral approach according to Bigliani. Extensive fracture exposure was avoided trying to perform the surgery as less invasive as possible [22]. The fracture was reduced by indirect maneuvers, with the help of $\mathrm{k}$-wires and/or an elevatorium or with bone hooks to reduce 


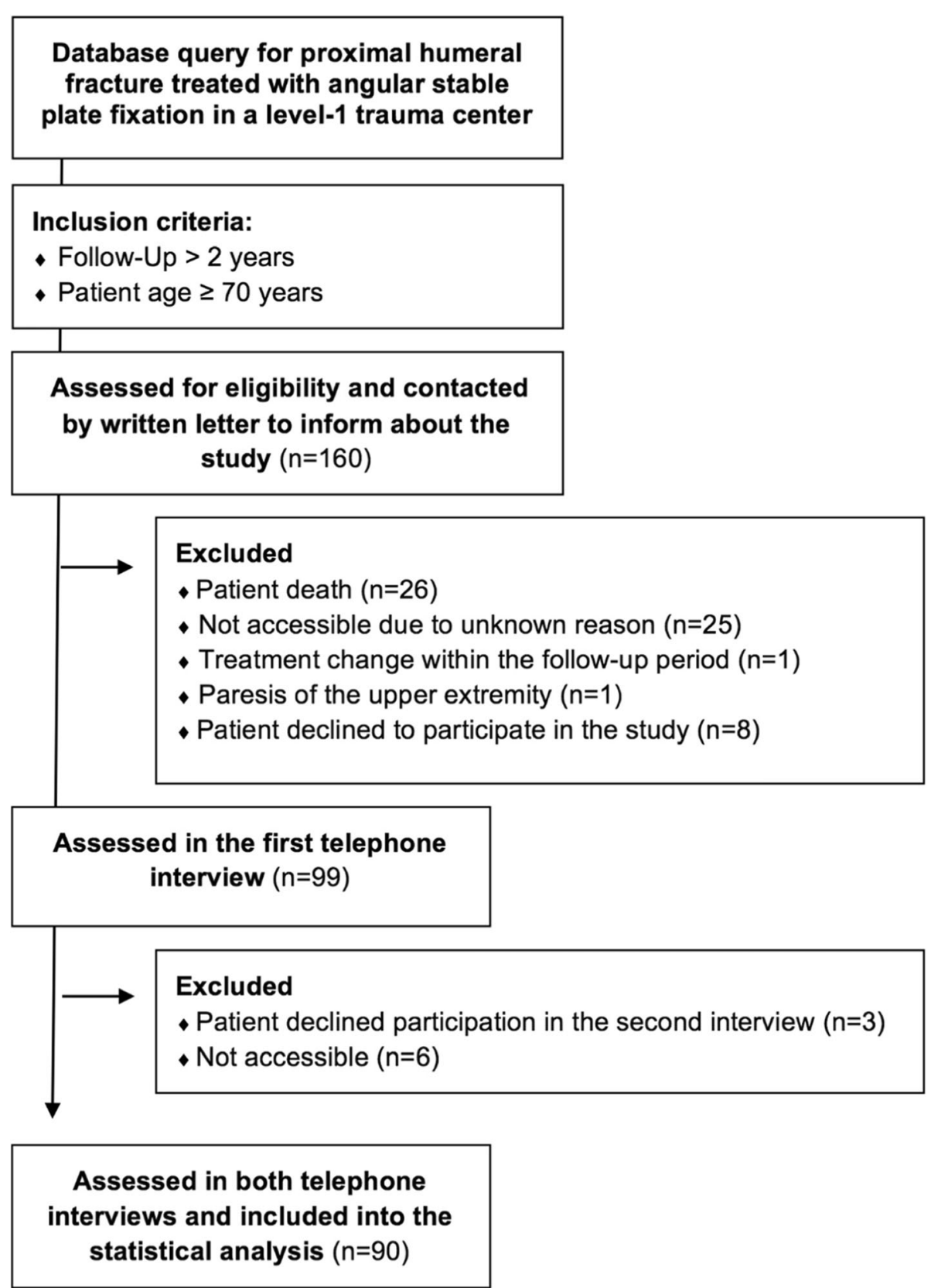

Fig. 1 Patient flow chart

the tuberosities. As described by Bahrs et al. [13], the plate was placed at least 5 to $8 \mathrm{~mm}$ distal to the upper end of the greater tuberosity and 2 to $4 \mathrm{~mm}$ lateral to the bicipital groove. Positioning of the plate and screws were controlled intraoperatively by an image intensifier.

Following surgery, the shoulder was immobilized using a shoulder sling during the first 7 days. Afterwards for 4 weeks, active-assisted movement including up to $90^{\circ}$ of abduction and flexion was allowed. All patients received physiotherapeutic treatment after the operation.

\section{Outcome variables}

The interview-based Constant score according to Boehm et al. [18], the DASH score, and the Oxford Shoulder Score were assessed during both telephone-based interviews. The Constant score assesses pain and shoulder function during daily activities, range of motion, and shoulder strength [23]. The adjusted Constant Score according to Boehm et al. [18] evaluates the strength of the shoulder with the help of housewares (e.g., water bottles, sugar packages) with a defined weight. These weights had to be held in front of the body without 
leaning against a wall in $90^{\circ}$ flexion in the shoulder and with an extended arm for at least $5 \mathrm{~s}$. The Constant score was categorized as excellent from 100 to 86 points, good from 71 to 85 points, satisfying from 70 to 56 points, and worse lower than 56 points [24]. The DASH score is a subject patient outcome score with 30 items regarding symptoms and functionality of the upper limb. The final score is calculated based on a standardized formula and ranges from 0 (no disability) to 100 (most severe disability) [25]. Score categories were excellent and good from 0 to 15 points, satisfying from 16 to 40 points, and a worse result with more than 40 points [26]. The OSS includes questions about pain, sleep, and daily life activities and ranges from 12 to 60 points. Outcome measured with the OSS is categorized in poor from 12 to 20 points, satisfying from 21 to 30 points, good from 31 to 40, and very good between 41 and 60 points [27].

\section{Statistical analysis}

The continuous scores were described descriptively as mean (median; range; minimum-maximum). Categories of scores, implant-related complications, as well as radiographic parameters were described as $n$ (\%). Shapiro-Wilk test was used to check if data was normally distributed. As scores were not normally distributed, correlations between the first and the second interview were computed with Spearman's rank correlation coefficient. The coefficients were analyzed using a scale proposed by Hopkins [28]: correlation coefficient $<0.1$, trivial relationship; 0.1-0.3, low; 0.30.5 , moderate; $0.5-0.7$, strong; $0.7-0.9$, very strong; > 0.9 , nearly perfect. Cross tabulation was applied and weighted Kappa measure of agreement $(k)$ was calculated to assess the test-retest agreement of the categories of each score. Weighting was done in accordance to the above described categories for each score: trichotomized $(0,0.5,1)$ DASH score, as well as quatrochotomized $(0,0.33,0.67,1)$ OSS and Constant score. Bland and Altman-analyses [29] were calculated to describe mean differences and limits of agreement between the two interviews for each score.
A two-sided $p$-value of $<0.05$ was considered statistically significant.

\section{Results}

Score values of the first and the second interview assessed by the physician assistant and the medical doctor are summarized in Table 1 . The OSS differed with a mean score of 55.7 points (median 58; range 33-60) in the first interview and 52.7 points (median 55; range 2560 points) in the second interview. The mean DASH score was 14.7 points (median 12.5; range $0-64.2$ ) in the first interview and 19.6 points (median 18.3; range 0 $66.7)$ in the second interview. The mean adjusted Constant score according to Boehm et al. [18] was 86.5 (median 91; range 40-100) points in the first interview and 64.2 (median 65.5; range 23-86) points in the second interview.

The categories of the individual scores are displayed in Fig. 2. The figure shows that the majority of patients reached in the first interview of the Constant score an excellent result $(n=55 ; 61 \%)$ and in the second interview a good result $(n=34 ; 38 \%)$. In the OSS, nearly all patients had a very good result in the first $(n=87 ; 97 \%)$ and second $(n=86 ; 96 \%)$ interview. The category of the DASH score was in the majority of patients 'excellent/ good' in the first interview and 'satisfying' in the second interview.

\section{Test-retest agreement}

The correlations between test and retest were strong to very strong. The correlation coefficients (Spearman) were $r=0.67(p<0.0001)$ for the Constant score, $r=0.77$ $(p<0.0001)$ for the DASH, and $r=0.71(p<0.0001)$ for the OSS. The agreement of categories between the first and the second interview were $55.9 \%$ (weighted $k=0.08$ ) for the Constant score, $87.2 \%$ (weighted $k=0.62$ ) for the DASH score, and 99.3\% (weighted $k=0.74$ ) for the OSS (all $p<0.001$ ). Bland-Altman analyses (see Fig. 3) showed significant systematic changes in scores indicated by 95\%CI which did not include 0. Moreover, limits of agreement of all three scores, especially for the Constant

Table 1 Summary of score assessments (mean (median; range)). A negative value of individual differences between interviews means that the score was lower in the second interview

\begin{tabular}{|c|c|c|c|c|c|c|}
\hline & $\begin{array}{l}\text { First score } \\
\text { assessment }\end{array}$ & $\begin{array}{l}\text { Second score } \\
\text { assessment }\end{array}$ & $\begin{array}{l}\text { Individual differences } \\
\text { between interviews }\end{array}$ & $\begin{array}{l}\text { Spearman's } \\
\text { correlation }\end{array}$ & $\begin{array}{l}\text { Weighted } k \text { measure of } \\
\text { agreement (categories) }\end{array}$ & $\begin{array}{l}\text { Agreement between } \\
\text { interviews (categories) }\end{array}$ \\
\hline OSS & $\begin{array}{l}55.7 \text { (median: } \\
\text { 58; 33-60) }\end{array}$ & $\begin{array}{l}52.7 \text { (median: } \\
55 ; 25-60)\end{array}$ & $\begin{array}{l}-3.0 \text { (median: }-2 ;-17- \\
5)\end{array}$ & 0.71 & 0.74 & $99.3 \%$ \\
\hline DASH & $\begin{array}{l}14.7 \text { (median: } \\
12.5 ; 0-64.2 \text { ) }\end{array}$ & $\begin{array}{l}19.6 \text { (median: } \\
\text { 18.3; 0-66.7) }\end{array}$ & $\begin{array}{l}4.9 \text { (median: } 2.5 ;-14.2- \\
31.7 \text { ) }\end{array}$ & 0.77 & 0.62 & $87.2 \%$ \\
\hline Constant & $\begin{array}{l}86.5 \text { (median: } \\
91 ; 40-100)\end{array}$ & $\begin{array}{l}64.2 \text { (median: } \\
65.5 ; 23-86)\end{array}$ & $\begin{array}{l}-22.3 \text { (median: }-21 ;- \\
53-10)\end{array}$ & 0.67 & 0.08 & $55.9 \%$ \\
\hline
\end{tabular}




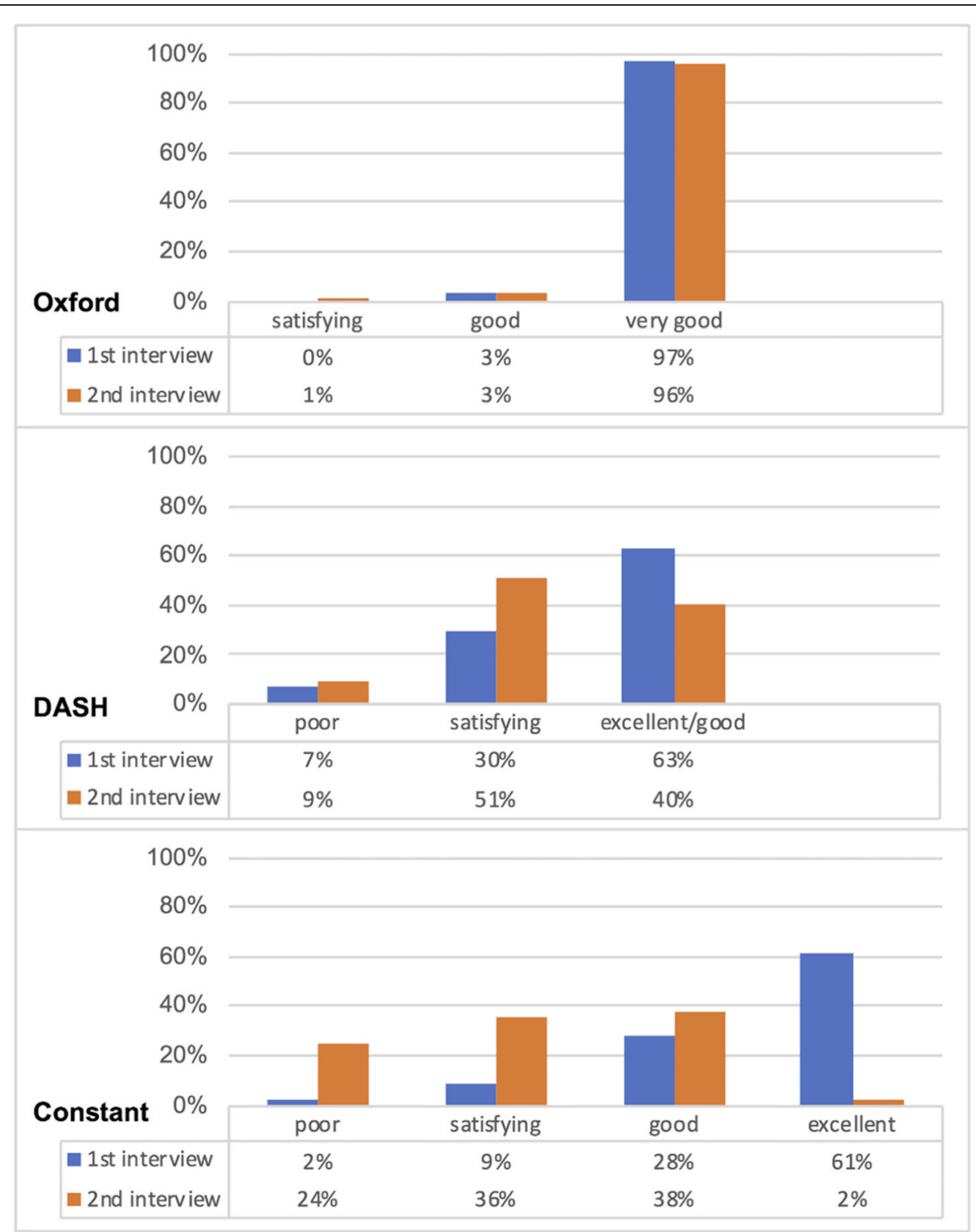

Fig. 2 Changes of the categorized results of the Oxford, DASH, and Constant score between the first (blue) and second (orange) interview

score, showed large ranges indicating low individual score agreement.

\section{Radiographic evaluation}

Anatomic reduction postoperatively was achieved in 55 (63.2\%) cases. Twenty-four (27.6\%) patients had either a displacement of the tuberosity, a varus/valgus angulation on the AP view of $\pm 15^{\circ}$, or an anteversion/retroversion on the axillary view of $\pm 15^{\circ}$. Eight $(9.2 \%)$ patients had a displacement of the tuberosity and/or a varus/valgus angulation and an anteversion/retroversion postoperatively.

\section{Discussion}

The primary aim of the present study was to describe patient-reported and clinical outcomes following angular stable plate fixation of proximal humeral fractures in patients over 70 years of age. As hypothesized, most patients showed good to excellent subjective outcomes at least two years following surgical treatment. Moreover, retrospective analysis of postoperative radiographs documented satisfying results with sufficient reduction of the fracture in most of the patients.

Most published studies analyzed short-term outcomes or reported about cohorts with patients younger than 70 years old. In the meta-analysis by Dai et al. [30], included studies reported Constant scores between 65.2 and 83.9 points between 6 and 60 months following locking compression plate fixation of proximal humeral fractures [31, 32]. Ockert et al. [14] reported about 43 fractures with an average age of 67.6 year and a median 


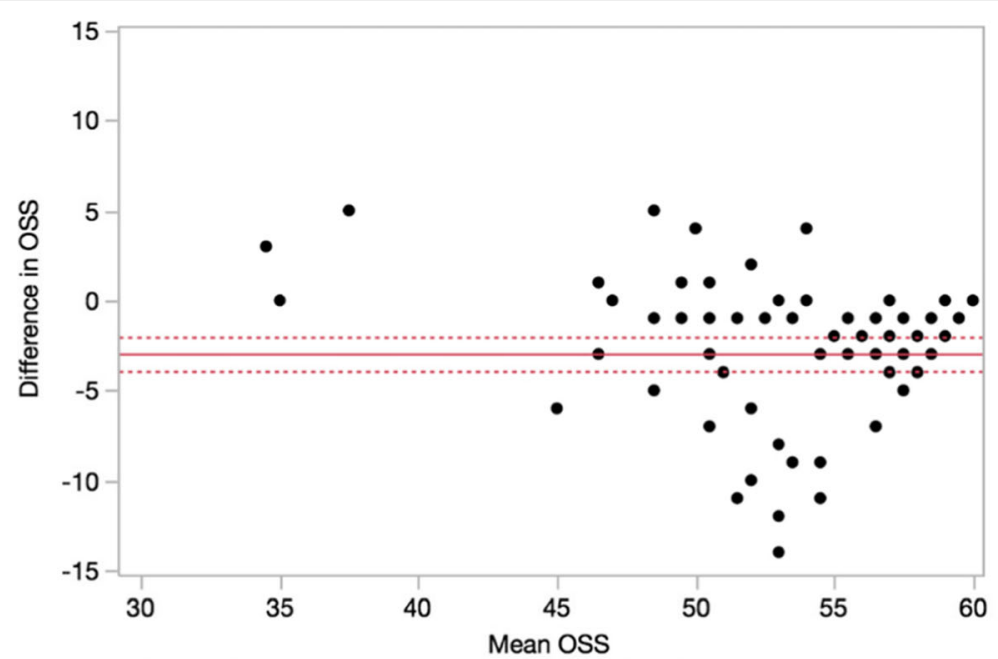

Mean difference: -2.97 (Cl -3.9 to -2.0$)$; Limits of agreement: -11.9 to 6.0 )

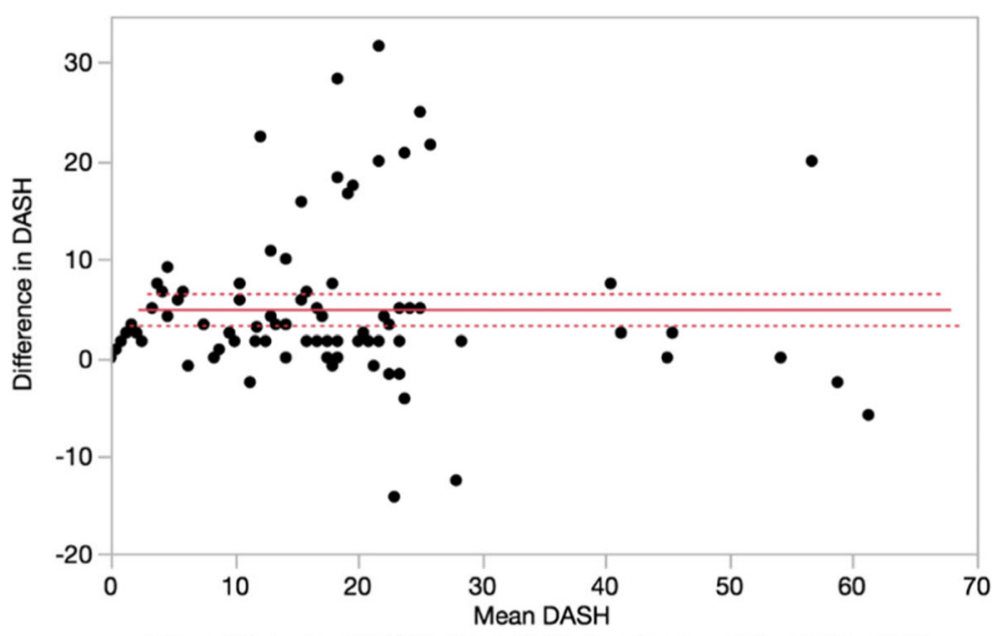

Mean difference: 4.9 (CI 3.3 to 6.5$)$; Limits of agreement: -10.6 to 20.4$)$

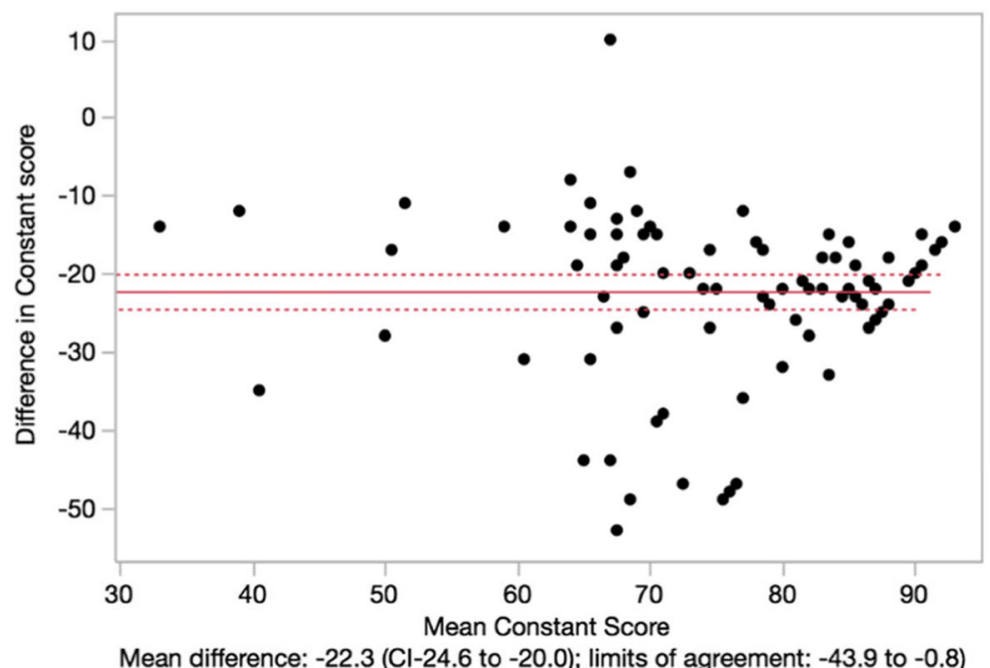

Fig. 3 Bland-Altman analyses showing plots with 95\% Cl and 95\% limits of agreement of the Oxford shoulder score (OSS), the DASH score, and the Constant score 
follow-up of 10 years. Patients reached 75.3 points in mean in the Constant score and the majority showed good to excellent outcomes. However, $16 \%$ of the patients had a poor long-term outcome [14]. Fifty-seven patients aged $65 \pm 14$ years were analyzed by Hirschmann et al. [33]. Four to 6 years postoperatively, patients reached $15.3 \pm 17.7$ points in the DASH score and $70.5 \pm 17.7$ points in the Constant score. In a prospective evaluation of 77 patients (mean age, 62 years) with a mean follow-up period of 96 months, Bahrs et al. [13] found a mean Constant and DASH score of 79 points and 12 points. Schulte et al. [34] found in 43 patients (average age, 68 years) with proximal humeral fractures treated with a locking compression plate a mean DASH score of 11 points (range $0-21.7$ ). Plath et al. [35] compared patients with a proximal humeral fracture older than 60 years who were treated with a locking blade nail or a locking plate (PHILOS). One year postoperatively, patients treated with a locking plate reached a median Constant score of $64 \pm 20$ points and a DASH score of $42 \pm 19$ points.

A poor functional outcome as well as higher risk of screw-related complications following plate fixation is highly related to age and gender [13, 14] $[36,37]$. Our sample consisted of patients older than 70 years with a mean age of 78.1 years. In the first and second assessment of our study, the Constant score was 86.5 points and 64.2 points, the DASH score 14.7 and 19.6 points, and 55.7 and 52.7 points in the OSS. Despite the older sample of the present study, our results underlined the benefits of surgical intervention. Scores showed similar values as studies with younger patients [33, 38].

The secondary aim of our study was to evaluate the assessment of telephone-based interviewing in a blinded test-retest analysis. As hypothesized, the agreement of the telephone-based assessments of the functional impairment reported in the interview-adjusted Constant score is low. The agreement between categories (55.9\%) was limited as well as high mean individual absolute differences between the interviews. These differences of the Constant score were mostly related to different answers about pain free mobilization as well as measurement of muscle strength. Both categories account for $65 \%$ of the total score. Subjective parameter such as pain and daily life activities account for smaller proportions. The measurement of muscle strength and mobilization was adapted by the interview-adjusted Constant score according to Boehm et al. [18]. It was reported as a reliable and valid tool to assess the clinical outcome in patients who cannot attend a follow-up appointment. However, this requires high compliance of patients and accurate instructions by the interviewer.

We could only find small differences between the first and the second assessment of the DASH and the OSS.
Despite higher test-retest agreements, both scores do not analyze the objective functionality and mobility of the shoulder as the Constant score. Additionally, the Bland-Altman method revealed that also the DASH and the OSS were limited in the absolute individual score agreement. Limits of agreement represent possible differences of approximately $15.5 \%$ and $9.0 \%$ of their respective scoring scales. However, high agreement between the categories of the DASH $(87.2 \%)$ and the OSS (99.3\%) were found for these scores.

Previously published by Mahabier et al. [16], the validity and reliability of outcome evaluation over time was reported for the DASH and the Constant score in humeral shaft fractures. The sample consisted of $140 \mathrm{pa}-$ tients with a median age of 58 years during a 1-year postoperative interval. In accordance to our results, the reliability of the DASH score was higher than for the Constant Score [16]. Slobogean et al. [39] found smaller mean differences in the Bland-Altman analysis for the DASH score (mean difference, 0.4 (95\% CI -2.3 to 3.1$)$ ) compared to our study. However, similar limits of agreement ( -15.2 to 15.9 ) were found. In our study, interviews were conducted from two different observers: a physical assistant and a medical doctor. Differences between the scores could have also been influenced by inter-observer differences of the education, personality, motivation, and how detailed instructions were made [40]. However, using two observers guaranteed blinded data collection. Results are limited as no inter-observer reliability was assessed.

Further limitations of the present study relied on the study design. Telephone-based interviews are a useful alternative to evaluate and monitor surgical outcomes, especially the DASH and the OSS, and to detect possible complications with low costs, easier and faster assessment of the interview, and lower lost-to-follow-up rates. Our follow-up rate $(56.3 \%)$ was higher compared to the follow-up rates of previous studies, e.g., Bahrs et al. [13] (40\%) or Ockert et al. [14] (35\%). Patient's death was the main reason of lost-to-follow-up. Despite lower lost-tofollow-up rates, the assessment of functional outcomes is difficult via phone calls, especially in the elderly population and answers of patients could not be verified. Following our study results, we do not recommend using the CS in a telephone-based assessment of shoulder function in elderly patients. An additional appointment in our clinic could have addressed this issue and would have decreased the re-call bias. Interview skills and differences in interview assessment can highly influence interview results. We used standardized scores which were validated in several studies. To reduce differences occurred by the different interviewer, we paid attention to strictly stick to a standardized interview protocol (order of questions etc.). Both interviewers were 
educated by the same senior consultant who supervised five telephone-based interviews, each.

In our study, the interviews were conducted from two different observers at two different time points: a physical assistant and a medical doctor. Differences between the scores could have also been influenced by interobserver differences of the education, personality, motivation, and how detailed instructions were made. However, using two observers guaranteed blinded data collection. Results are limited as no inter-observer reliability was assessed.

\section{Conclusion}

Most patients reported about good subjective outcomes at least 2 years following surgical treatment. The test-retest agreement of the clinical outcomes measured with an interview-based CS was low. Only small differences and high agreement between score categories were found for DASH and OSS. Despite higher test-retest agreement, both scores do not analyze the functionality and mobility of the shoulder as detailed as the Constant score. Telephone-based assessment of OSS and DASH presents as an alternative to collect and monitor surgical outcomes in elderly patients with low costs, easier assessment, and lower lost-to-follow-up rates.

\section{Abbreviations}

AO: Arbeitsgemeinschaft für Osteosynthesefragen; DASH: Disabilities of the Arm, Shoulder and Hand; OSS: Oxford Shoulder Score

\section{Acknowledgments}

We acknowledge support by Deutsche Forschungsgemeinschaft and Open Access Publishing Fund of University of Tübingen.

\section{Research registration number}

250/2011BO2.

\section{Disclaimer}

No financial bias existed. The local Ethical Committee (University of Tübingen) approved the study (83250/2011BO2).

\section{Authors' contributions}

$P Z, K S, C B$, and MA have initiated the study, have made the data collection, and interpretation. MA and KS have made the statistics. PZ and MA have written the manuscript and have analyzed most of the data. All authors read and approved the final manuscript.

\section{Funding}

There is no funding source.

\section{Availability of data and materials}

The datasets used and analyzed during the current study are available from the corresponding author on reasonable request.

\section{Ethics approval and consent to participate}

Informed consent was obtained from all individual participants included in the study. The study was conducted according to the Helsinki Declaration (Ethical Principles for Medical Research Involving Human Subjects) and was approved by the University of Tuebingen ethics committee.

\section{Consent for publication}

Not applicable.

\section{Competing interests}

The authors declare that they have no competing interests.

\section{Author details}

${ }^{1}$ BG- Center for Trauma \& Reconstructive Surgery, Eberhard Karls University of Tübingen, 72076 Tübingen, Germany. ${ }^{2}$ AO Research Institute Davos, 7270

Davos, Switzerland.

Received: 30 October 2019 Accepted: 20 December 2019

Published online: 06 January 2020

\section{References}

1. Bahrs C, Bauer M, Tepass A, Weise K, Rolauffs B, Bahrs SD, Eingartner C, Blumenstock $\mathrm{G}$. The complexity of proximal humeral fractures is age and gender specific. J Orthop Sci. 2013;18:465-70. https://doi.org/10.1007/ s00776-013-0361-x.

2. Court-Brown CM, Garg A, McQueen MM. The epidemiology of proximal humeral fractures. Acta Orthop Scand. 2001;72:365-71. https://doi.org/10. 1080/000164701753542023.

3. Kannus P, Palvanen M, Niemi S, Sievänen H, Parkkari J. Rate of proximal humeral fractures in older Finnish women between 1970 and 2007. Bone. 2009:44:656-9. https://doi.org/10.1016/j.bone.2008.12.007.

4. Passaretti D, Candela V, Sessa P, Gumina S. Epidemiology of proximal humeral fractures: a detailed survey of 711 patients in a metropolitan area. J Shoulder Elb Surg. 2017;26:2117-24. https://doi.org/10.1016/j.jse.2017.05.029.

5. Sun Q, Ge W, Li G, Wu J, Lu G, Cai M, Li S. Locking plates versus intramedullary nails in the management of displaced proximal humeral fractures: a systematic review and meta-analysis. Int Orthop. 2018;42:641-50.

6. Kancherla VK, Singh A, Anakwenze OA. Management of acute proximal humeral fractures. JAAOS-J Am Acad Orthop Surg. 2017;25:42-52.

7. Handoll HH, Brorson S (2015) Interventions for treating proximal humeral fractures in adults Cochrane database Syst rev:CD000434. doi: https://doi. org/10.1002/14651858.CD000434.pub4.

8. Bell JE, Leung BC, Spratt KF, Koval KJ, Weinstein JD, Goodman DC, Tosteson $\mathrm{AN}$. Trends and variation in incidence, surgical treatment, and repeat surgery of proximal humeral fractures in the elderly. J Bone Joint Surg Am. 2011;93:121-31. https://doi.org/10.2106/JBJS.I.01505.

9. Huttunen $\Pi$, Launonen AP, Pihlajamaki H, Kannus P, Mattila VM. Trends in the surgical treatment of proximal humeral fractures-a nationwide 23-year study in Finland. BMC Musculoskelet Disord. 2012;13:261. https://doi.org/10. 1186/1471-2474-13-261.

10. Robinson CM, Stirling PH, Goudie EB, MacDonald DJ, Strelzow JA. Complications and long-term outcomes of open reduction and plate fixation of proximal humeral fractures. JBJS. 2019;101:2129-39.

11. Helwig P, Bahrs C, Epple B, Oehm J, Eingartner C, Weise K. Does fixed-angle plate osteosynthesis solve the problems of a fractured proximal humerus? A prospective series of 87 patients. Acta Orthop. 2009;80:92-6.

12. Roderer G, Erhardt J, Kuster M, Vegt P, Bahrs C, Kinzl L, Gebhard F. Second generation locked plating of proximal humerus fractures-a prospective multicentre observational study. Int Orthop. 2011;35:425-32. https://doi.org/ 10.1007/s00264-010-1015-7.

13. Bahrs C, Kühle L, Blumenstock G, Stöckle U, Rolauffs B, Freude T. Which parameters affect medium-to long-term results after angular stable plate fixation for proximal humeral fractures? J Shoulder Elb Surg. 2015;24:727-32. https://doi.org/10.1016/j.jse.2014.08.009.

14. Ockert B, Siebenburger G, Kettler M, Braunstein V, Mutschler W. Long-term functional outcomes (median 10 years) after locked plating for displaced fractures of the proximal humerus. J Shoulder Elb Surg. 2014;23:1223-31. https://doi.org/10.1016/j.jse.2013.11.009.

15. Osterhoff G, Hoch A, Wanner GA, Simmen H-P, Werner CM. Calcar comminution as prognostic factor of clinical outcome after locking plate fixation of proximal humeral fractures. Injury. 2012;43:1651-6. https://doi. org/10.1016/j.injury.2012.04.015

16. Mahabier KC, Den Hartog D, Theyskens N, Verhofstad MHJ, Van Lieshout EMM, Investigators HT. Reliability, validity, responsiveness, and minimal important change of the disabilities of the arm, shoulder and hand and Constant-Murley scores in patients with a humeral shaft fracture. J Shoulder Elb Surg. 2017;26:e1-e12. https://doi.org/10.1016/j.jse.2016.07.072.

17. Davidson M, Keating J. Patient-reported outcome measures (PROMs): how should I interpret reports of measurement properties? A practical guide for 
clinicians and researchers who are not biostatisticians. Br J Sports Med. 2014:48:792-6. https://doi.org/10.1136/bjsports-2012-091704.

18. Boehm D, Wollmerstedt N, Doesch M, Handwerker M, Mehling E, Gohlke F. Development of a questionnaire based on the Constant-Murley-score for self-evaluation of shoulder function by patients. Unfallchirurg. 2004;107:397402. https://doi.org/10.1007/s00113-004-0757-3.

19. Agha RA, Borrelli MR, Vella-Baldacchino M, Thavayogan R, Orgill DP, Group S. The STROCSS statement: strengthening the reporting of cohort studies in surgery. Int J Surg. 2017;46:198-202. https://doi.org/10.1016/j.jisu.2017.08.586.

20. Neer CS 2nd. Displaced proximal humeral fractures. I. Classification and evaluation. J Bone Joint Surg Am. 1970;52:1077-89.

21. Müller M, Nazarian $S$, Koch P, Schatzker J. The comprehensive classification of fractures of long bones, vol. 43. Berlin, Heidelberg: Springer Science \& Business Media; 1990.

22. Hessmann M, Baumgaertel F, Gehling H, Klingelhoeffer I, Gotzen L. Plate fixation of proximal humeral fractures with indirect reduction: surgical technique and results utilizing three shoulder scores. Injury. 1999;30:453-62.

23. Yian EH, Ramappa AJ, Arneberg O, Gerber C. The constant score in normal shoulders. J Shoulder Elb Surg. 2005;14:128-33. https://doi.org/10.1016/j.je. 2004.07.003.

24. Bahrs C, Badke A, Rolauffs B, Weise K, Zipplies S, Dietz K, Eingartner C. Longterm results after non-plate head-preserving fixation of proximal humeral fractures. Int Orthop. 2010;34:883-9. https://doi.org/10.1007/s00264-0090848-4.

25. Hudak PL, Amadio PC, Bombardier C, Beaton D, Cole D, Davis A, Hawker G, Katz JN, Makela M, Marx RG. Development of an upper extremity outcome measure: the DASH (disabilities of the arm, shoulder, and head). Am J Ind Med. 1996;29:602-8

26. Angst F, Schwyzer HK, Aeschlimann A, Simmen BR, Goldhahn J. Measures of adult shoulder function: Disabilities of the Arm, Shoulder, and Hand Questionnaire (DASH) and its short version (QuickDASH), Shoulder Pain and Disability Index (SPADI), American Shoulder and Elbow Surgeons (ASES) Society standardized shoulder assessment form, Constant (Murley) Score (CS), Simple Shoulder Test (SST), Oxford Shoulder Score (OSS), Shoulder Disability Questionnaire (SDQ), and Western Ontario Shoulder Instability Index (WOSI). Arthritis Care Res. 2011;63(Suppl 11):S174-88. https://doi.org/ 10.1002/acr.20630

27. Bahrs C, Oehm J, Rolauffs B, Eingartner C, Weise K, Dietz K, Helwig P. T-plate osteosynthesis--an obsolete osteosynthesis procedure for proximal humeral fractures? Middle-term clinical and radiological results. Zeitschrift fur Orthopadie und Unfallchirurgie. 2007;145:186-94.

28. Hopkins WG (1997) A new view of statistics. Will G. Hopkins. http://sportsci. org/resource/stats/. Accessed 2016 Feb 012016.

29. Bland JM, Altman D. Statistical methods for assessing agreement between two methods of clinical measurement. Lancet. 1986;327:307-10.

30. Dai J, Chai Y, Wang C, Wen G. Meta-analysis comparing locking plate fixation with hemiarthroplasty for complex proximal humeral fractures. Eur J Orthop Surg Traumatol. 2014;24:305-13. https://doi.org/10.1007/s00590-0131179-0.

31. Spross C, Platz A, Erschbamer M, Lattmann T, Dietrich M. Surgical treatment of Neer group VI proximal humeral fractures: retrospective comparison of PHILOS and hemiarthroplasty. Clin Orthop Relat Res. 2012;470(7):2035. https://doi.org/10.1007/s11999-011-2207-1.

32. Zhang JH, Di ZL, He ZY, Feng JX, Xu RM. Comparison of humeral head replacement and internal fixation for the treatment of 3 parts and 4 parts fractures of proximal humerus in the elderly. Zhongguo Gu Shang. 2010;23: 435-9.

33. Hirschmann MT, Fallegger B, Amsler F, Regazzoni P, Gross T. Clinical longerterm results after internal fixation of proximal humerus fractures with a locking compression plate (PHILOS). J Orthop Trauma. 2011;25:286-93. https://doi.org/10.1097/BOT.0b013e3181f2b20e.

34. Schulte LM, Matteini LE, Neviaser RJ. Proximal periarticular locking plates in proximal humeral fractures: functional outcomes. J Shoulder Elb Surg. 2011; 20:1234-40. https://doi.org/10.1016/j.jse.2010.12.015.

35. Plath JE, Kerschbaum C, Seebauer T, Holz R, Henderson DJH, Förch S, Mayr E. Locking nail versus locking plate for proximal humeral fracture fixation in an elderly population: a prospective randomised controlled trial. BMC Musculoskelet Disord. 2019;20:20. https://doi.org/10.1186/s12891-019-2399-1.

36. Balcells-Diaz E, Daunis IEP. Shoulder strength value differences between genders and age groups. J Shoulder Elb Surg. 2017;27(3):463. https://doi. org/10.1016/j.jse.2017.10.021.
37. Panagiotopoulou VC, Varga P, Richards RG, Gueorguiev B, Giannoudis PV. Late screw-related complications in locking plating of proximal humerus fractures: a systematic review. Injury. 2019;50:2176-95. https://doi.org/10. 1016/j.injury.2019.11.002.

38. Solberg BD, Moon CN, Franco DP, Paiement GD. Locked plating of 3- and 4part proximal humerus fractures in older patients: the effect of initial fracture pattern on outcome. J Orthop Trauma. 2009;23:113-9. https://doi. org/10.1097/BOT.0b013e31819344bf.

39. Slobogean GP, Noonan VK, O'Brien PJ. The reliability and validity of the disabilities of arm, shoulder, and hand, EuroQol-5D, health utilities index, and short form-6D outcome instruments in patients with proximal humeral fractures. J Shoulder Elb Surg. 2010;19:342-8. https://doi.org/10.1016/j.jse. 2009.10.021.

40. Salazar MK. Interviewer bias: how it affects survey research. AAOHN J. 1990; 38:567-72.

\section{Publisher's Note}

Springer Nature remains neutral with regard to jurisdictional claims in published maps and institutional affiliations.

Ready to submit your research? Choose BMC and benefit from:

- fast, convenient online submission

- thorough peer review by experienced researchers in your field

- rapid publication on acceptance

- support for research data, including large and complex data types

- gold Open Access which fosters wider collaboration and increased citations

- maximum visibility for your research: over $100 \mathrm{M}$ website views per year

At BMC, research is always in progress.

Learn more biomedcentral.com/submissions 